\title{
Renormalization group flow of quartic perturbations in graphene: Strong coupling and large- $N$ limits
}

\author{
Joaquín E. Drut and Dam Thanh Son \\ Institute for Nuclear Theory, University of Washington, Seattle, WA 98195-1550, USA
}

(Dated: October 25, 2018)

\begin{abstract}
We explore the renormalization group flow of quartic perturbations in the lowenegy theory of graphene, in the strong Coulomb coupling and large- $N$ limits, where $N$ is the number of fermion flavors. We compute the anomalous dimensions of the quartic couplings $u$ up to leading order in $1 / N$ and find both relevant and irrelevant directions in the space of quartic couplings. We discuss possible phase diagrams and relevance for the physics of graphene.
\end{abstract}

PACS numbers: 73.63.Bd, 05.10.Cc

\section{INTRODUCTION}

Graphene, a single layer of graphite, has lately attracted increasing attention, especially since its experimental realization [1]. This material presents a number of unusual electronic features, such as a Landau level structure that gives rise to half-integer quantum Hall effect. In general, those properties can be traced back to the low-energy spectrum, governed by two-component massless fermions that come in four flavors: two due to electronic spin and two from degenerate Dirac points in the band structure [2]. The quasi-relativistic electronic spectrum is characterized by a velocity $v \simeq c / 300 \ll c$, where $c$ is the speed of light. However, the Coulomb interaction, which is instantaneous for all practical purposes, breaks this emergent Lorentz invariance. In particular, the electron velocity becomes scaledependent [3].

It was noted recently [4] that a generalizaton of the low-energy theory that describes graphene possesses a quantum critical point. Reference 4 considered a model of $N$ species of $2+1$ dimensional two-component massless Dirac fermions interacting through a 3D instantaneous Coulomb interaction with a coupling constant $g$. It was then shown, by analyzing the renormalization group $(\mathrm{RG})$ flow of the fermion velocity, that for sufficiently large $N$, the strongly coupled limit $g^{2} N / v \rightarrow \infty$ is a quantum critical point, characterized by a dynamic critical exponent $z=1-8 /\left(\pi^{2} N\right)+O\left(1 / N^{2}\right)$. It was also argued that real graphene is close to this critical point for a large momentum window.

In the present work we further explore the quantum critical properties of this theory by studying the RG flow of various quartic perturbations of the Thirring [5] and Gross-Neveu [6] varieties. These four-fermion interactions are irrelevant at weak coupling, but obtain nontrivial anomalous dimensions of order $1 / N$ at the strong coupling fixed point. If a four- 
fermi coupling becomes relevant below some $N$, one may expect dynamical gap generation, in which case the system becomes an insulator, or that the system would flow to another fixed point.

The question of whether the Coulomb interaction makes graphene an insulator has been considered previously. In Ref. 7 it was found from solving the gap equation with a screened Coulomb interaction that, for $N<8 / \pi \approx 2.55$, a gap opens at sufficiently large coupling. For $N>8 / \pi$ the system remain gapless at all couplings. This was confirmed in Ref. 8 using a similar approach. However, as the approximations employed in these works are uncontrolled, one would like to explore alternative approaches to this problem. The RG analysis in this paper is one of them. (Instability toward ferromagnetism has also been considered [9].)

We implement a Wilson-Fisher RG transformation and find the anomalous dimensions of the various couplings. Throughout the paper the Coulomb parameter $\lambda=g^{2} N / 32 v$ is kept finite and the limit $\lambda \rightarrow \infty$ is discussed at the end. In this limit we identify both relevant and irrelevant directions in the parameter space of the quartic couplings and discuss possible scenarios, including gap generation and the the flow to a new non-gaussian fixed point. At the end of our results section we address the relevance of our findings for real graphene.

The paper is organized as follows: the model and Feynman rules are presented in section II, the details of the calculation are outlined in section III, the results are discussed in section IV and the conclusions presented in section V.

\section{MODEL AND FEYNMAN RULES}

Consider a model of $N / 2$ flavors of four-component relativistic fermions (or $N$ flavors of two-component fermions) $\psi_{a}$ interacting through an instantaneous Coulomb interaction and through generic $\mathrm{U}(N / 2)$-symmetric quartic interactions. The corresponding Euclidean action is

$$
\begin{gathered}
S_{E}=-\int d t d^{2} x\left(\bar{\psi}_{a} \gamma^{0} \partial_{0} \psi_{a}+v \bar{\psi}_{a} \gamma^{i} \partial_{i} \psi_{a}+i A_{0} \bar{\psi}_{a} \gamma^{0} \psi_{a}\right)+\frac{1}{2 g^{2}} \int d t d^{3} x\left(\partial_{i} A_{0}\right)^{2} \\
+\frac{1}{2 N} \int d t d^{2} x\left[u_{1}\left(\bar{\psi}_{a} \psi_{a}\right)^{2}+u_{2}\left(\bar{\psi}_{a} \gamma_{0} \gamma_{i} \psi_{a}\right)^{2}+u_{3}\left(\bar{\psi}_{a} \gamma_{0} \gamma_{5} \psi_{a}\right)^{2}+u_{4}\left(\bar{\psi}_{a} \gamma_{0} \psi_{a}\right)^{2}\right. \\
\left.+u_{5}\left(\bar{\psi}_{a} \gamma_{i} \psi_{a}\right)^{2}+u_{6}\left(\bar{\psi}_{a} \gamma_{5} \psi_{a}\right)^{2}\right]
\end{gathered}
$$

where the $\gamma^{\mu}, \mu=0,1,2$ (we shall also use Latin indices for the spatial directions) are Dirac matrices satisfying a Euclidean Clifford algebra $\left\{\gamma^{\mu}, \gamma^{\nu}\right\}=2 \delta^{\mu \nu}$, and $\gamma^{5}=\gamma^{0} \gamma^{1} \gamma^{2}$. We can choose, for example, a representation of this algebra in which

$$
\gamma^{0}=\left(\begin{array}{cc}
\sigma^{3} & 0 \\
0 & -\sigma^{3}
\end{array}\right), \quad \gamma^{i}=\left(\begin{array}{cc}
\sigma^{i} & 0 \\
0 & -\sigma^{i}
\end{array}\right),
$$

where $\sigma^{i}$ are the Pauli matrices. In the real world $N / 2=2$, corresponding to two spin polarizations of the electrons. The two two-component spinors near the two valleys are combined 
into a four-component spinor. The action (2.1) is invariant under spin rotations, but is not invariant under rotations in the valley space. ${ }^{1}$ Notice that the four-fermi terms are rotationally invariant, but not Lorentz invariant: the latter is broken by the Coulomb interaction. Apart from the four-fermi terms written in (2.1), one can also introduce an independent set equal to $\gamma^{3}$ times the vertices already included, where $\gamma^{3}$ is linearly independent from the other $\gamma$-matrices and $\left\{\gamma^{3}, \gamma^{\mu}\right\}=0$. One possible choice is

$$
\gamma^{3}=\left(\begin{array}{ll}
0 & \mathbb{1} \\
\mathbb{1} & 0
\end{array}\right) .
$$

Such matrices, together with the ones already considered, form a complete basis for the algebra of $4 \times 4$ Dirac matrices. We restrict for the moment to the action (2.1), but we shall comment on these extra vertices in our discussion section.

We shall use the large- $N$ limit to do calculations at large values of the coupling $g$, so $N$ will remain arbitary (but large) until the end. Notice that, unlike the fermionic degrees of freedom, the Coulomb field $A_{0}$ lives in $3+1$ dimensions, which is reflected in its kinetic term. In the strong coupling $g \rightarrow \infty$ limit this term disappears and the $A_{0}$ propagator is dominated by quantum corrections coming from the fermion loops, as we shall see below.
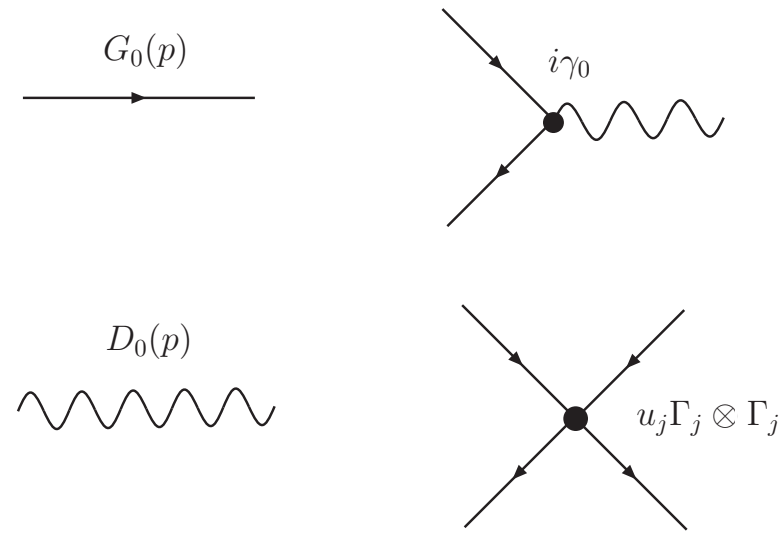

FIG. 1: Feynman rules (see text for details).

In $2+1$ dimensions the naive dimensions of the fields and couplings are as follows: $[\psi]=m,\left[A_{0}\right]=m,[g]=m^{0},\left[u_{j}\right]=m^{-1}$.

The Feynman rules are as in Fig. 1, where the fermion propagator is

$$
G_{0}(p)=\frac{i \not p}{p^{2}}
$$

where $p=\left(p_{0}, \vec{p}\right), \vec{p}$ being a 2D momentum vector. Here and in the rest of the paper, we use the notation $\not p=\gamma^{0} p_{0}+v \vec{\gamma} \cdot \vec{p}$ and $p^{2}=p_{0}^{2}+v^{2}|\vec{p}|^{2}$.

\footnotetext{
${ }^{1}$ The two degenerate Dirac points in the band structure of graphene are sometimes referred to as valleys. Thus, rotations in valley space correspond to operations that mix the upper and lower components of the four-component Dirac fermions of our theory.
} 
The boson-fermion interaction vertex is $i \gamma_{0}$, and the quartic vertices are $-\frac{u_{j}}{N} \Gamma_{j} \otimes \Gamma_{j}$, with $\Gamma_{j} \in\left\{1, \gamma_{0} \gamma_{i}, \gamma_{0} \gamma_{5}, \gamma_{0}, \gamma_{i}, \gamma_{5}\right\}$

Finally, the bare boson $\left(A_{0}\right)$ propagator is

$$
D_{0}(p)=\frac{g^{2}}{2|\vec{p}|}
$$

In the large $N$, finite $g^{2} N$ limit we must resum the fermion loop contributions to this propagator (see Fig. 2).

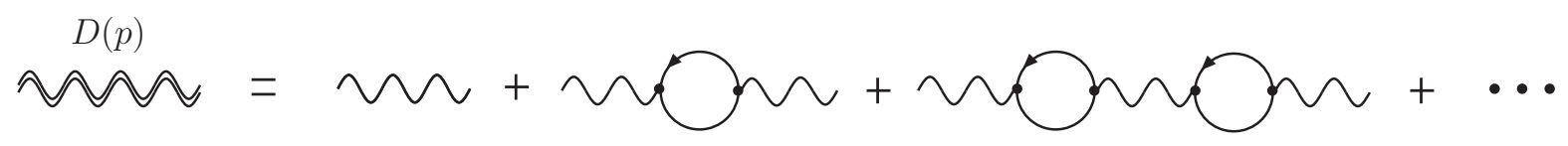

FIG. 2: Resummation of the one-loop self-energy contribution to the boson propagator.

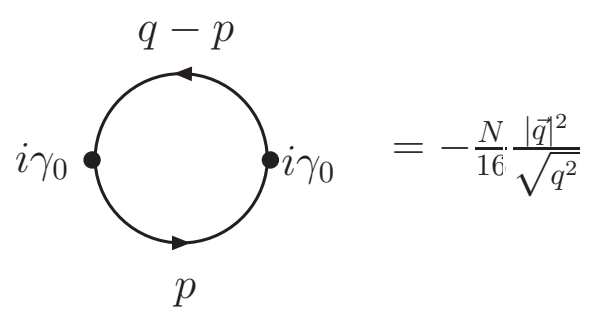

FIG. 3: One-loop self-energy contribution to the boson propagator.

Such resummation (which is equivalent to the random phase approximation) results in a dressed boson propagator that we shall use in the rest of this work, as shown in Figs. 2 and 3 :

$$
D(q)=\left(\frac{2|\vec{q}|}{g^{2}}+\frac{N}{16} \frac{|\vec{q}|^{2}}{\sqrt{q^{2}}}\right)^{-1}=\frac{16}{N} \frac{\sqrt{q^{2}}}{|\vec{q}|^{2}}\left(1+\frac{1}{\lambda} \frac{\sqrt{q^{2}}}{|\vec{q}|}\right)^{-1}=\frac{g^{2}}{2|\vec{q}|}\left(1+\lambda \frac{|\vec{q}|}{\sqrt{q^{2}}}\right)^{-1}
$$

where $\lambda=g^{2} N /(32 v)$.

\section{CALCULATION OF THE RG FLOW}

We implement a Wilsonian RG procedure whereby we integrate out modes in the momentum shell $\Lambda_{1}<p<\Lambda_{0}$ and then rescale the coordinates as well as the fields. The RG equation has the form

$$
\frac{\partial \mathbf{u}(p)}{\partial \ln p}=(\mathbb{1}-\mathbf{M}(\lambda)) \mathbf{u}(p)
$$

where $[\mathbf{u}]_{i}=u_{i}$ and $\mathbf{M}(\lambda)$ is a $6 \times 6$ matrix. The $\mathbb{1}$ in the right-hand side comes from the naive dimension of $u_{i}$. Furthermore

$$
\mathbf{M}(\lambda)=\mathbf{M}_{v}(\lambda)+\mathbf{M}_{w f}(\lambda),
$$


where $\mathbf{M}_{v}$ comes from the vertex corrections and $\mathbf{M}_{w f}$ comes from wavefunction renormalization.

\section{A. Vertex renormalization}

To the first nontrivial order in $1 / N$, finding the RG flow of $u_{j}$ entails computing the

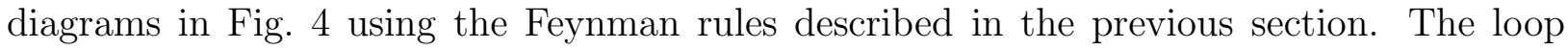
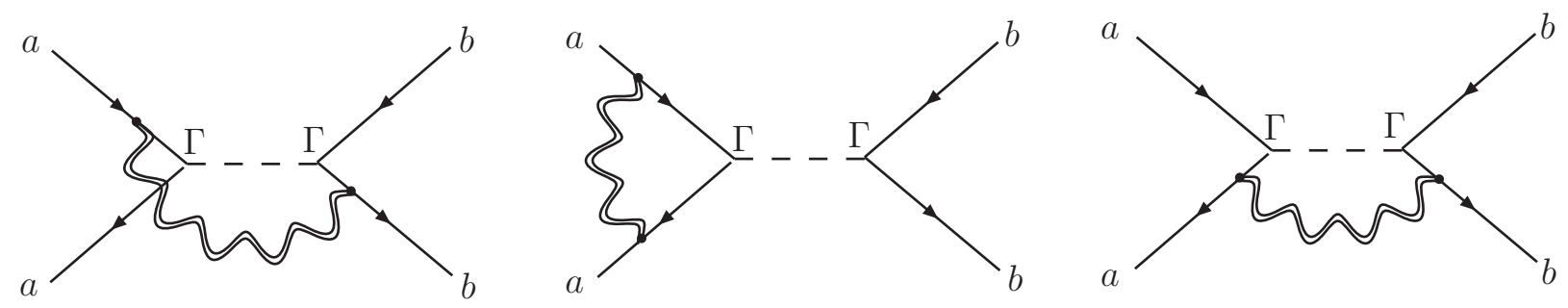

FIG. 4: Contributions to the RG flow of $u_{j}$ coming from vertex renormalization. $a$ and $b$ are flavor indices and $\Gamma$ is a generic four-Fermi vertex. The dashed line is intended to show the difference between the loops in the various diagrams. Exchange of dummy indices leads to the same diagrams and results in an overall factor of 2 .

integrals have the same form for all three diagrams, namely

$$
\begin{aligned}
& \frac{16}{N} \int_{\Lambda_{1}}^{\Lambda_{0}} \frac{d^{3} q}{(2 \pi)^{3}} \frac{q^{\mu} q^{\nu}}{q^{4}} \frac{\lambda}{|\vec{q}|}\left(1+\lambda \frac{|\vec{q}|}{\sqrt{q^{2}}}\right)^{-1}=\frac{4}{N \pi^{2}} \ln \left[\frac{\Lambda_{0}}{\Lambda_{1}}\right] \times I_{0}(\lambda) \quad \text { for } \mu=\nu=0 \\
& \times \frac{I_{1}(\lambda)}{2} \text { for } \mu=\nu=i
\end{aligned}
$$

and vanish for $\mu \neq \nu$. The integration region is spherically symmetric. Furthermore, notice that the integrand is independent of the azimuthal angle, and that the remaining radial and angular integrals are decoupled because $|\vec{q}|=\sqrt{q^{2}} \sqrt{1-t^{2}}$, where $t=\cos \theta$ and $\theta$ is the polar angle, making the calculation straightforward. We have used the following definitions:

$$
\begin{gathered}
I_{0}(\lambda)=\int_{-1}^{1} d t \frac{t^{2}}{\sqrt{1-t^{2}}} \frac{\lambda}{1+\lambda \sqrt{1-t^{2}}}=-2+\frac{\pi}{\lambda}+\frac{2 \sqrt{\lambda^{2}-1}}{\lambda} \ln \left[\lambda+\sqrt{\lambda^{2}-1}\right] \\
I_{1}(\lambda)=\int_{-1}^{1} d t \frac{\lambda \sqrt{1-t^{2}}}{1+\lambda \sqrt{1-t^{2}}}=2-\frac{\pi}{\lambda}+\frac{2}{\lambda \sqrt{\lambda^{2}-1}} \ln \left[\lambda+\sqrt{\lambda^{2}-1}\right]
\end{gathered}
$$

In the large $\lambda$ limit, $\lambda \rightarrow \infty$,

$$
I_{0}(\lambda) \rightarrow-2+2 \ln \left[\lambda+\sqrt{\lambda^{2}-1}\right]
$$

while

$$
I_{1}(\lambda) \rightarrow 2
$$


The divergence of $I_{0}(\lambda \rightarrow \infty)$ is associated the the fact that the Coulomb interaction is unscreened at zero momentum and nonzero frequency. As we shall see, however, all such divergences cancel out in the $\beta$ functions for $u_{i}$.

With the above identities at hand it is not very difficult to compute the contributions of the diagrams in Fig. 4 to the $\beta$ functions. The only significant step that remains to be discussed is straightforward Dirac algebra to find the products of a small number of $\gamma$ matrices (coming from the vertices and the fermion propagators). For instance, for $\Gamma_{4}=\gamma_{0}$, the calculation of the diagram in the middle of Fig. 4 entails computing

$$
\begin{aligned}
\gamma_{0} \gamma_{\mu} \gamma_{0} \gamma_{\nu} \gamma_{0} & =\gamma_{0} & & \text { for } \mu=\nu=0 \\
& =-\gamma_{0} & & \text { for } \mu=\nu=i
\end{aligned}
$$

where we only need the case $\mu=\nu$ because the integral (3.3) is otherwise zero. The contribution of this diagram to the flow of $u_{4}$ is therefore

$$
\Delta u_{4}=u_{4} \frac{8}{N \pi^{2}} \ln \left[\frac{\Lambda_{0}}{\Lambda_{1}}\right]\left(I_{0}-I_{1}\right)
$$

where we have included the factor of 2 coming from an essentially identical diagram where the boson propagator appears connecting the fermion lines on the right instead of on the left. For this specific vertex the first and third diagrams in Fig. 4 cancel out exactly due to a sign difference in one of the fermion lines. In this particular case only diagonal flow is generated, i.e. this vertex does not contribute to the flow of the others. In general, however, there is operator mixing: each vertex contributes to its own flow but also to other vertices.

Our result turns out to be

$$
\mathbf{M}_{v}(\lambda)=\frac{8}{N \pi^{2}}\left(\begin{array}{cccccc}
I_{0}+I_{1} & -2 I_{1} & 0 & 0 & 0 & 0 \\
-I_{1} & I_{0} & 0 & 0 & 0 & 0 \\
0 & 0 & I_{0}-I_{1} & 0 & 0 & 0 \\
0 & 0 & 0 & I_{0}-I_{1} & 0 & 0 \\
0 & 0 & 0 & 0 & I_{0} & -I_{1} \\
0 & 0 & 0 & 0 & -2 I_{1} & I_{0}+I_{1}
\end{array}\right)
$$

\section{B. Wavefunction renormalization}

Upon integration of the high-momentum degrees of freedom, new coordinates and fields are defined, that are rescaled versions of the original ones. Doing this explicitly results in the following action:

$$
S_{\text {eff }}=-\int d t^{\prime} d^{2} x^{\prime}\left\{Z_{0}^{-1} b_{1}^{-2} \bar{\psi}_{a} \gamma^{0} \partial_{0}^{\prime} \psi_{a}+Z_{1}^{-1} b_{1}^{-1} b_{0}^{-1} \bar{\psi}_{a} \gamma^{i} \partial_{i}^{\prime} \psi_{a}+\ldots\right\}
$$

where the subscript "eff" indicates that this action describes modes with momenta $|p|<$ $\Lambda_{1}=b_{1} \Lambda_{0}$. Here $t^{\prime}=b_{0} t x^{\prime}=b_{1} x, 0<b_{0}, b_{1}<1$, and $\ldots$ contains all the terms in the action that are not quadratic in the fermion fields. We shall renormalize the field as

$$
\psi_{a} \rightarrow \psi_{a}^{\prime}=Z_{0}^{-1 / 2} b_{1}^{-1} \psi_{a}
$$


and enforce the nonrenormalization of the fermion velocity by requiring

$$
\frac{Z_{1}^{-1} b_{1}^{-1} b_{0}^{-1}}{Z_{0}^{-1} b_{1}^{-2}}=\frac{Z_{0} b_{1}}{Z_{1} b_{0}}=1
$$

which means that the integration shells should actually be non-spherical and have radii related by

$$
b_{0}=\frac{Z_{0}}{Z_{1}} b_{1}
$$

However $Z_{0} / Z_{1}=1+O(1 / N)$, therefore the deviation from spherical symmetry is small and is not important to the order of $1 / N$ we are considering. Thus we can compute the loop integral, assuming that the integration region is spherically symmetric.

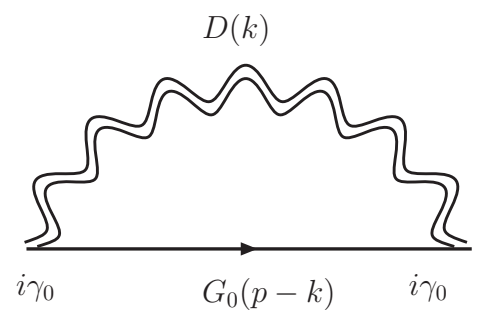

FIG. 5: One-loop self-energy correction to the fermion propagator.

The one-loop self-energy correction to the inverse fermion propagator was computed in Ref. 4. In our notation, it corresponds to

$$
\begin{aligned}
& Z_{0}^{-1}=1-\Delta Z_{0}=1+\frac{4}{N \pi^{2}}\left[I_{0}(\lambda)-I_{1}(\lambda)\right] \ln \left[\frac{\Lambda_{0}}{\Lambda_{1}}\right] \\
& Z_{1}^{-1}=1-\Delta Z_{1}=1+\frac{4}{N \pi^{2}} I_{0}(\lambda) \ln \left[\frac{\Lambda_{0}}{\Lambda_{1}}\right]
\end{aligned}
$$

The correction to the fermion propagator is suppressed by $1 / N$, which is another reason to work in the large $N$ limit. Without this small parameter the diagram of Fig. (5) would be of order one.

To see how the wavefunction renormalization affects the running of the four-fermi coupling, we write down a generic quartic term in the action

$$
u \int d t d^{2} x\left(\sum_{a=1}^{N} \bar{\psi}_{a} \Gamma \psi_{a}\right)^{2},
$$

Rescaling of the momenta and the fields as discussed above leads to a renormalization of the coupling constant $u$ according to

$$
u \rightarrow u^{\prime}=b_{1}^{-2} b_{0}^{-1}(u+\Delta u)\left(Z_{0}^{-1 / 2} b_{1}^{-1}\right)^{-4}=b_{1}\left(u+\Delta u+u\left(\Delta Z_{0}+\Delta Z_{1}\right)\right)+O\left(u^{2} / N\right)
$$

where we have also included the contribution $\Delta u$ from the vertex renormalization, and where $\Delta Z_{0}+\Delta Z_{1}$ comes from wavefunction renormalization. 
From Eqs. (3.16) and (3.17) we find

$$
\mathbf{M}_{w f}(\lambda)=-\frac{4}{N \pi^{2}}\left[2 I_{0}(\lambda)-I_{1}(\lambda)\right]
$$

\section{RESULTS AND DISCUSSION}

Combining Eqs. (3.11) and (3.20), we find

$$
\mathbf{M}(\lambda)=\frac{4}{N \pi^{2}} I_{1}(\lambda)\left(\begin{array}{cccccc}
3 & -4 & 0 & 0 & 0 & 0 \\
-2 & 1 & 0 & 0 & 0 & 0 \\
0 & 0 & -1 & 0 & 0 & 0 \\
0 & 0 & 0 & -1 & 0 & 0 \\
0 & 0 & 0 & 0 & 1 & -2 \\
0 & 0 & 0 & 0 & -4 & 3
\end{array}\right)
$$

Notice that the terms containing $I_{0}(\lambda)$ cancel out, as anticipated, so the limit $\lambda \rightarrow \infty$ is finite. The eigenvalues yield the anomalous dimensions: $\gamma_{a}=-\frac{8}{N \pi^{2}}[5,-1,-1,-1,-1,5]$. The two operators with lowest anomalous dimensions, $-40 / N \pi^{2}$, are

$$
2\left(\bar{\psi}_{a} \psi_{a}\right)^{2}-\left(\bar{\psi}_{a} \gamma_{0} \gamma_{i} \psi_{a}\right)^{2}, \quad 2\left(\bar{\psi}_{a} \gamma_{5} \psi_{a}\right)^{2}-\left(\bar{\psi}_{a} \gamma_{i} \psi_{a}\right)^{2}
$$

Including the vertices of the form $\gamma^{3} \Gamma_{j} \otimes \gamma^{3} \Gamma_{j}$, that were mentioned in the introduction, one can repeat the calculation and obtain as a result a copy of the above matrix. This is simply a consequence of $\left\{\gamma^{3}, \gamma^{\mu}\right\}=0$. There is no mixing between these new vertices and the ones considered in our calculation. The list of the most relevant operators may then be extended to include

$$
2\left(\bar{\psi}_{a} \gamma^{3} \psi_{a}\right)^{2}-\left(\bar{\psi}_{a} \gamma^{3} \gamma_{0} \gamma_{i} \psi_{a}\right)^{2}, \quad 2\left(\bar{\psi}_{a} \gamma^{3} \gamma_{5} \psi_{a}\right)^{2}-\left(\bar{\psi}_{a} \gamma^{3} \gamma_{i} \psi_{a}\right)^{2}
$$

whose the anomalous dimensions are also $-40 / N \pi^{2}$.

In the large $N$ limit, the anomalous dimensions are small, hence all four-fermi operators have dimensions close to 4 and are irrelevant. However, as one decreases $N$ the dimensions of some operators decrease as well. Naively, at

$$
N<N_{\text {crit }}=\frac{40}{\pi^{2}} \approx 4.05
$$

the operators in (4.2) and (4.3) would have dimensions less than 3, and become relevant. Of course, this is only an extrapolation of our leading-order result to finite $N$. Nevertheless, it is quite interesting that $N_{\text {crit }}$ is close to the real-world value of $N=4$. (For comparison, for the Lorentz-invariant Thirring model the critical number of four-component Dirac fermions is quoted as 6.6(1) [10], to be compared with $N_{\text {crit }} / 2 \approx 2$.) Due to the limitation of our calculations, we cannot determine whether the exact $N_{\text {crit }}$ is smaller or larger than 4 .

If $N_{\text {crit }}<4$, then the physics at $N=4$ and infinite coupling is governed by the strongcoupling fixed point discussed in Ref. 4. If $N_{\text {crit }}>4$, then there are two further possibilities 
for $N=4, \lambda=\infty$. It may turn out that the system develops a gap, and becomes an insulator. In this case one expects a bifermion operator to have a nonzero expectation value, breaking a discrete symmetry. Unfortunately, simply from the form of the operators (4.2) and (4.3) it is not possible to conclude, with definiteness, which of the discrete symmetries will be spontaneously broken. Another possibility is that the coupling flows into a new stable fixed point (a similar situation was discussed in Ref. 11.) In this case the system remains gapless, but with a new dynamic critical behavior. This case is illustrated in Fig. 6, where we also show the $\beta$ function of the coupling $u$.

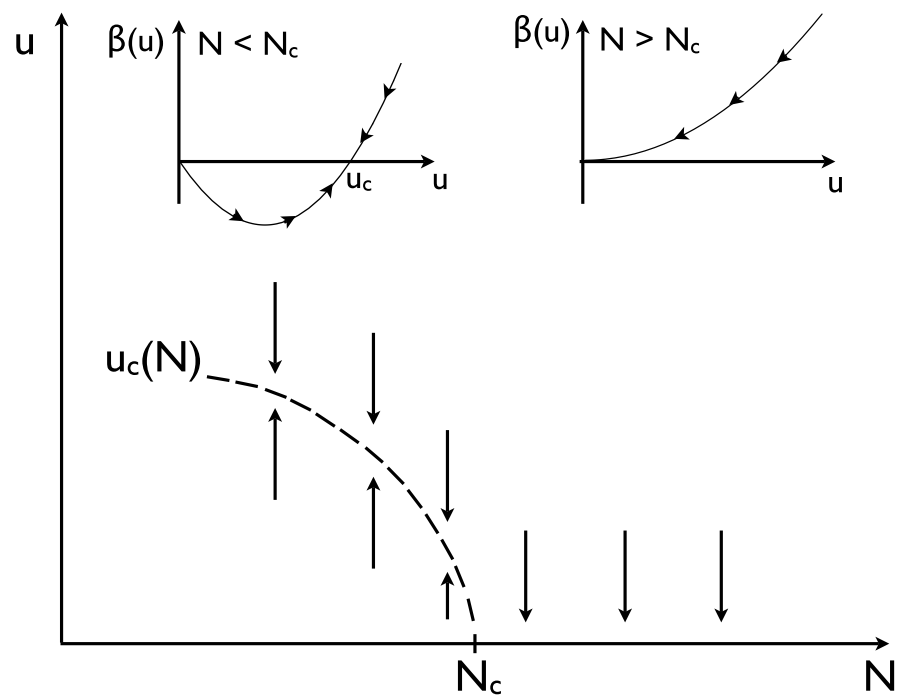

FIG. 6: Phase diagram and beta functions (Inset), in one particular scenario.

In real graphene, on the other hand, $\lambda$ is large but not infinite, so in the above discussion we should take into account that the anomalous dimensions are functions of both $N$ and $\lambda$. In real graphene one has

$$
\lambda=\frac{e^{2} N}{32 \epsilon_{0} \hbar v}
$$

where $N=4$ and $v=10^{6} \mathrm{~m} / \mathrm{s}$, so that the anomalous dimension of the operators (4.2) and (4.3) is $\gamma \approx-0.72$. Thus, all four-fermi operators are irrelevant, at least to the order of $1 / N$ we are considering. It is conceivable, however, that higher-order corrections will push $\gamma$ to be below -1 . On the other hand, for graphene on a $\mathrm{SiO}_{2}$ substrate with dielelectric constant $\epsilon=5.5$, the coupling $\lambda$ is reduced by a factor of $2 /(1+\epsilon)$, and $\gamma \approx-0.45$, substantially above -1 . In this case, one can conclude that the four-fermi interactions are irrelevant (and will become more irrelevant as the fermion velocity $v$ increases in the infrared). 


\section{CONCLUSION}

We have studied the RG flow of various four-Fermi couplings $u$ in the low-energy theory of graphene, generalized to include $N$ different fermion flavors. We computed the anomalous dimensions of the various couplings to the first nontrivial order in $1 / N$. In the limit of infinitely strong Coulomb interaction, the operators with lowest dimensions become relevant for $N<N_{\text {crit }}$, where $N_{\text {crit }}$ is estimated to be 4 . In real graphene with $N=4$ but at finite Coulomb coupling, the four-fermi interactions are irrelevant, at least to the leading nontrivial order in $1 / N$.

It would be interesting to further investigate the phase diagram of our model. One can try to push the calculations to another order in $1 / N$. In addition, one should try to perform numerical simulations of the model. The most interesting values of $N$ where nontrivial phases may exist, as indicated by our calculations, are lower values like $N=2$ and $N=4$.

\section{Acknowledgments}

This work is supported, in part, by DOE grant No. DE-FG02-00ER41132.

Note added: After this work was completed, the authors learned of Ref. 12, in which the running of four-fermi interactions is also considered. Our lowest anomalous dimension coincides with the value found in Ref. 12, but our values of other anomalous dimensions disagree with Ref. 12. We thank Igor Aleiner for bringing Ref. 12 to our attention.

[1] A. K. Geim and K. S. Novoselov, "The rise of graphene," Nat. Mat. 6, 183 (2007), and references therein.

[2] G. W. Semenoff, "Condensed matter simulation of a three-dimensional anomaly," Phys. Rev. Lett. 53, 2449 (1984).

[3] J. González, F. Guinea and M. A. H. Vozmediano, "Non-Fermi liquid behavior of electrons in the half-filled honeycomb lattice (A Renormalization group approach)," Nucl. Phys. B 424, 595 (1994) arXiv:hep-th/9311105.

[4] D. T. Son, "Quantum critical point in graphene approached in the limit of infinitely strong Coulomb interaction," Phys. Rev. B 75235423 (2007) arXiv:cond-mat/0701501].

[5] W. Thirring, "A soluble relativistic field theory", Ann. Phys. 3, 91 (1958).

[6] D. Gross and A. Neveu, "Dynamical symmetry breaking in asymptotically free field theories", Phys. Rev. D 103235 (1974).

[7] E. V. Gorbar, V. P. Gusynin, V. A. Miransky, and I. A. Shovkovy, "Magnetic field driven metal-insulator phase transition in planar systems," Phys. Rev. B 66, 045108 (2002) arXiv:cond-mat/0202422]. 
[8] H. Leal and D. V. Khveshchenko, "Excitonic instability in two-dimensional degenerate semimetals, Nucl. Phys. B 687, 323 (2004) arXiv:cond-mat/0302164.

[9] N. M. R. Peres, F. Guinea, and A. H. Castro Neto, "Coulomb interactions and ferromagnetism in pure and doped graphene," Phys. Rev. B 72, 174406 (2005).

[10] S. Christofi, S. Hands, and C. Strouthos, "Critical flavor number in the three dimensional Thirring model." Phys. Rev. D 75, 101701(R) (2007) arXiv:hep-lat/0701016].

[11] I. F. Herbut, "Interactions and phase transitions on graphene's honeycomb lattice," Phys. Rev. Lett. 97146401 (2006).

[12] I. L. Aleiner, D. E. Kharzeev, and A. M. Tsvelik, "Spontaneous symmetry breakings in graphene subjected to in-plane magnetic field," Phys. Rev. B 76, 195415 (2007). 